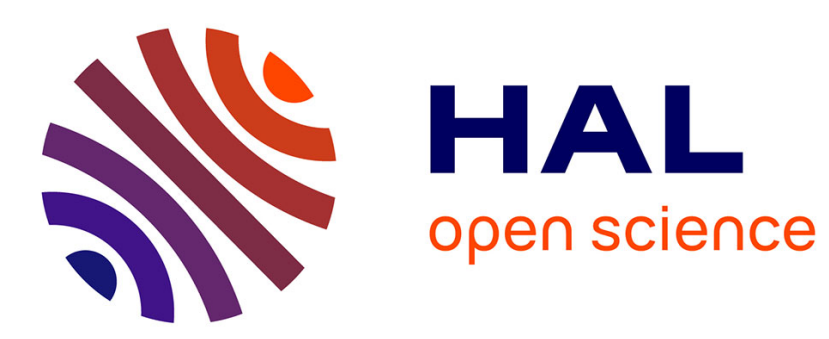

\title{
On the rigid projectile model for low velocity impacts
}

E. Jacquelin

\section{To cite this version:}

E. Jacquelin. On the rigid projectile model for low velocity impacts. International Journal of Impact Engineering, 2009, 36 (8), pp.1006. 10.1016/j.ijimpeng.2009.01.002 . hal-00587977

\section{HAL Id: hal-00587977 https://hal.science/hal-00587977}

Submitted on 22 Apr 2011

HAL is a multi-disciplinary open access archive for the deposit and dissemination of scientific research documents, whether they are published or not. The documents may come from teaching and research institutions in France or abroad, or from public or private research centers.
L'archive ouverte pluridisciplinaire HAL, est destinée au dépôt et à la diffusion de documents scientifiques de niveau recherche, publiés ou non, émanant des établissements d'enseignement et de recherche français ou étrangers, des laboratoires publics ou privés. 


\section{Accepted Manuscript}

Title: On the rigid projectile model for low velocity impacts

Authors: E. Jacquelin

PII: $\quad$ S0734-743X(09)00024-4

DOI: $\quad$ 10.1016/j.jijmpeng.2009.01.002

Reference: IE 1745

To appear in: International Journal of Impact Engineering

Received Date: 17 July 2008

Revised Date: 25 November 2008

Accepted Date: 22 January 2009

Please cite this article as: Jacquelin E. On the rigid projectile model for low velocity impacts, International Journal of Impact Engineering (2009), doi: 10.1016/j.jijmpeng.2009.01.002

This is a PDF file of an unedited manuscript that has been accepted for publication. As a service to our customers we are providing this early version of the manuscript. The manuscript will undergo copyediting, typesetting, and review of the resulting proof before it is published in its final form. Please note that during the production process errors may be discovered which could affect the content, and all legal disclaimers that apply to the journal pertain. 


\title{
On the rigid projectile model for low velocity
}

\section{impacts}

\author{
E. Jacquelin ${ }^{\mathrm{a}}$

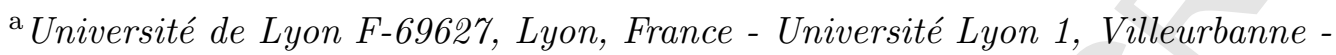 \\ INRETS, UMR-T 9406, LBMC - IUT, 17 rue de France - 69627 Villeurbanne - \\ France
}

tel: 33(0)472692132 - fax: 33(0)472692120

\begin{abstract}
Most of the studies about low velocity impact aimed to determine the impact force and the impact duration. The influence of the interaction law between the projectile and the target on the response has been examined; the influence of the structure model on the response has been addressed as well. However when the projectile is much more rigid than the structure, it has always been considered as an infinitely rigid mass. This paper shows that such a model tends to overestimate the maximum impact force and underestimate the impact duration; however, a 2-degree-of-freedom (dof) model has been suitable to recover the impact force very well. Moreover, it is shown that this rigid projectile may be viewed as a rigid mass associated with a modified Hertz law. These results have been established for a sphere-sphere impact and a sphere-beam impact as well. Nevertheless, it has been
\end{abstract}


shown that the 1-dof model is definitively relevant to determine the structural response: in fact the displacement of the structure provided by the 1-dof model and the 2-dof model are the same, as indicated in the last section.

Key words: structure modelling / Hertz law / antioscillator / rigid mass / impact

\section{Introduction}

Impact on flexible structures is a complex phenomenon which includes the interaction between the projectile and the structure, the response of the structure and the response of the projectile. A complete modelling of an impact event can always be done using a 3D Finite Element model. This method involves the consideration of the interface/contact between the impacting bodies: that increases the time required for numerical simulation, besides the use of powerful computers available nowadays. So it may be preferable to use simplified analytical models [1], [2], [3], [4] for calculating the contact force history; the interaction is very often modelled as a non-linear spring (stiffness $k_{H}$ ) describing the contact behaviour. Hertz law is the most widely used law [5], [6].

Likewise, the structures can always be modelled by the finite element method: if the structures are complex or discretized with a very fine mesh, the numerical cost will be very high. In order to decrease the number of degrees of freedom (dof) involved in the simulations, the modal description of the structures has been used [5], [6]. Nevertheless, the modal expansion is slowly convergent: hence a lot of eigenmodes would be required for a good accuracy. That is why the "anti-oscillators" have been defined by Jacquelin [7], [8] and used in the impact event simulations [9].

Email address: eric.jacquelin@univ-lyon1.fr (E. Jacquelin). 


\section{ARTICLE IN PRESS}

As to the projectile model, when the first eigenfrequencies (except the null rigid body frequencies) have been much higher than the structure ones, a 1-dof system has been usually considered [2], [3] to evaluate the contact force: the projectile is modelled by a rigid mass. The interaction laws and the structure models have been extensively studied in the literature whereas, to the author's knowledge, the assessment of that 1-dof model was much less addressed. So this paper aimed to assess the influence of the projectile model in an impact event, for a projectile far more rigid than the structure.

In fact, this study requires to achieve the antioscillator (AO) model of the studied structures. So, in the following, a short presentation of the anti-oscillators is first given: details of the method may be found in [7] and [8]; then, a sphere-sphere impact is studied to address the rigid mass model validity; finally the influence of the projectile model in a structure-sphere impact is examined.

\section{The antioscillator model}

The mass $\left(\mathbf{M}^{F E}\right)$ and stiffness $\left(\mathbf{K}^{F E}\right)$ matrices of the finite element model are supposed to be known; $n$ is the model dof number, $\mathbf{X}(t)$ is the dof vector and $\mathbf{F}(t)$ is the load vector. The AO description is very useful when one dof plays a particular role in the response of the structure: for example the dof associated with the impact location and direction when an impact event occurs. The AOs depend on the choice of one dof, $i_{0}$.

The AO model is based on a Ritz description that require to define the "static mode" and the "constraint modes". First, some specific matrices and vectors which depend on the chosen dof $i_{0}$ have to be defined: 


\section{ARTICLE IN PRESS}

- the AO mass $\mathbf{M}^{A O}$ (resp. AO stiffness $\mathbf{K}^{A O}$ ) matrix: this matrix is derived from the finite element mass $\mathbf{M}^{F E}$ (resp. stiffness $\mathbf{K}^{F E}$ ) matrix by replacing the $i_{0}^{\text {th }}$ row and the $i_{0}^{\text {th }}$ column by a row and a column filled with zeros;

- the static load $\mathbf{F}_{s t}$ : it is a $n$-size vector such as all of its elements are zero except the $i_{0}^{t h}$ element, $\mathbf{F}_{s t, i_{0}}$ :

$$
\left\{\begin{array}{l}
\mathbf{F}_{s t, i \neq i_{0}}=0 \\
\mathbf{F}_{s t, i_{0}}=1
\end{array}\right.
$$

The following "modes" can then be defined:

- The constraint modes $\left\{\boldsymbol{\phi}_{i}, \omega_{A R i}\right\}_{i=1 . . n-1}$ are the solution of the eigenproblem associated with the $\mathbf{M}^{A O}$ mass matrix and the $\mathbf{K}^{A O}$ stiffness matrix; hence the eigenvectors $\left\{\boldsymbol{\Phi}_{i}\right\}_{i=1 . . n-1}$ cancel out for the dof $i_{0}$ (see Fig. 1). Accordingly, the associated eigenfrequencies belong to the set of the antiresonant frequencies.

- The static mode $\boldsymbol{\phi}_{s t}$ is a dof vector such as:

$$
\mathbf{K}^{F E} \boldsymbol{\phi}_{s t}=c_{s t} \mathbf{F}_{s t}
$$

which has its $i_{0}^{\text {th }}$ element, $\boldsymbol{\phi}_{s t, i 0}$, equals to unity (see Fig. 1):

$$
\boldsymbol{\phi}_{s t, i 0}=1
$$

$c_{s t}$ is a normalisation constant chosen to normalise $\boldsymbol{\phi}_{s t}$ as indicated by Eq. (3).

- The residual mode $\boldsymbol{\phi}_{0}$ is a vector which verifies:

$$
\begin{aligned}
\boldsymbol{\phi}_{0} & =\boldsymbol{\phi}_{s t}-\sum_{i=1}^{n-1} c_{i} \boldsymbol{\phi}_{i} \\
\forall i \geq 1, \quad \boldsymbol{\phi}_{0}^{T} \mathbf{M}^{F E} \boldsymbol{\phi}_{i} & =0
\end{aligned}
$$




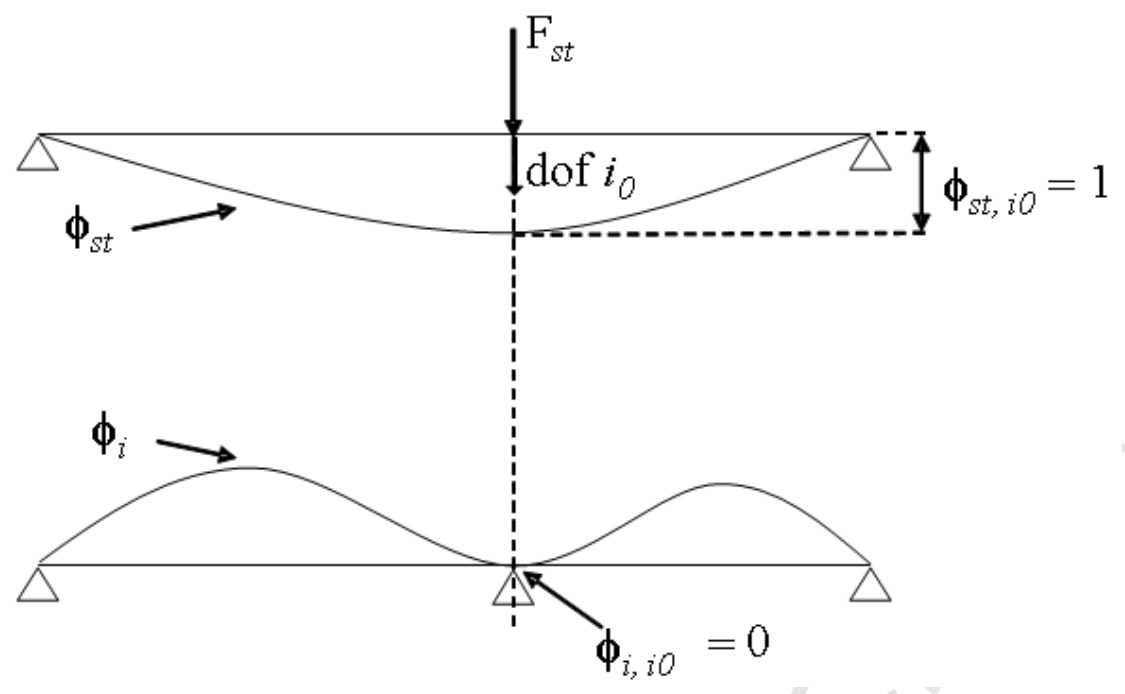

Fig. 1. Static and constraint mode

The orthogonality property (5) leads to:

$$
c_{i}=\frac{\boldsymbol{\phi}_{i}^{T} \mathbf{M}^{F E} \boldsymbol{\phi}_{s t}}{\boldsymbol{\phi}_{i}^{T} \mathbf{M}^{F E} \boldsymbol{\phi}_{i}}
$$

It must be emphasized that these modes depend on the structure and on the choice of dof $i_{0}$ but they don't depend on any loading. Accordingly, the static load is only a way to account for dof $i_{0}$ : the static load has no link with the actual loading of the structure.

Then, the dof vector $\mathbf{X}$ may be expanded in terms of the following Ritz-vectors:

$$
\mathbf{X}(t)=\sum_{i=0}^{n-1} q_{i}(t) \boldsymbol{\phi}_{i}
$$

The dof vector expansion (7) may be reorganized:

$$
\mathbf{X}(t)=\lambda_{0}(t) \boldsymbol{\psi}_{0}+\sum_{i=1}^{n-1}\left(\lambda_{i}(t)-\lambda_{0}(t)\right) \boldsymbol{\psi}_{i}
$$

where

$$
\begin{array}{rr}
\lambda_{0}(t)=q_{0}(t)=\mathbf{X}_{i_{0}} & \boldsymbol{\psi}_{0}=\boldsymbol{\phi}_{s t} \\
\lambda_{i}(t)=\frac{q_{i}(t)}{c_{i}} & \boldsymbol{\psi}_{i}=c_{i} \boldsymbol{\phi}_{i}
\end{array}
$$




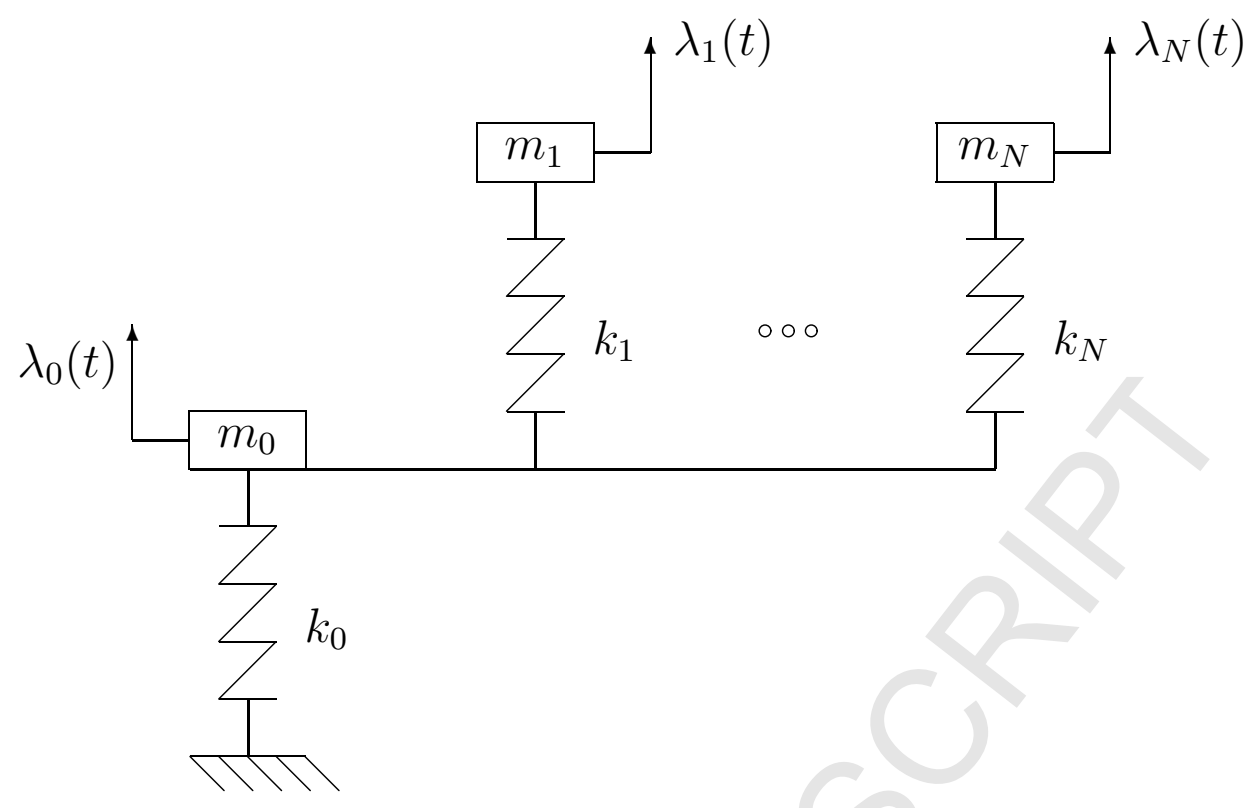

Fig. 2. AO model of any structure

In fact, the parameter $\lambda_{0}$ is the $i_{0}^{\text {th }}$ dof, $\mathbf{X}_{i_{0}}$.

This change of variables leads to the striking model represented Fig. 2 where the dof are the parameters $\left\{\lambda_{i}\right\}_{i=0 . . n-1}$ : the first single dof system $\left(m_{0}, k_{0}\right)$ is the base of the $n-1$ single dof systems $\left(m_{i}, k_{i}\right)_{i=1 . . n-1}$, called the antioscillators:

- $m_{i}$ :

$$
\begin{aligned}
\forall i \geq 1 \quad m_{i} & =c_{i}^{2} \boldsymbol{\phi}_{i}^{T} \mathbf{M}^{F E} \boldsymbol{\phi}_{i}=\frac{\left(\boldsymbol{\phi}_{i}^{T} \mathbf{M}^{F E} \boldsymbol{\phi}_{s t}\right)^{2}}{\boldsymbol{\phi}_{i}^{T} \mathbf{M}^{F E} \boldsymbol{\phi}_{i}} \\
m_{0} & =\boldsymbol{\phi}_{s t}^{T} \mathbf{M}^{F E} \boldsymbol{\phi}_{s t}-\sum_{i=1}^{n-1} m_{i}=m_{s t}-\sum_{i=1}^{n-1} m_{i}
\end{aligned}
$$

$m_{0}$ is referred to as the "residual mass"; $m_{s t}=\boldsymbol{\phi}_{s t}^{T} \mathbf{M}^{F E} \boldsymbol{\phi}_{s t}$ is the "static" mass.

- $k_{0}$ :

$$
k_{0}=\boldsymbol{\phi}_{s t}^{T} \mathbf{K}^{F E} \boldsymbol{\phi}_{s t}
$$

- For statically determinate structures, $k_{0}$ is the static stiffness.

- For statically indeterminate structures (i.e., $\boldsymbol{\phi}_{s t}$ is a rigid body eigenvector), $k_{0}=0$. 


\begin{tabular}{ccccc}
$E_{S}(\mathrm{GPa})$ & $\nu_{S}$ & $\rho_{S}\left(\mathrm{kgm}^{-3}\right)$ & $\operatorname{radius} R(\mathrm{~mm})$ & mass $(\mathrm{g})$ \\
\hline \multirow{2}{*}{210} & 0.3 & 7800 & 10 & 32.7 \\
\hline
\end{tabular}

Table 1: Properties of the sphere

- $k_{i}$ :

$$
\forall i \geq 1 \quad k_{i}=\omega_{A R i}^{2} m_{i}
$$

The relation (9) shows that the AO masses are independent of the norm of the eigenshapes $\left\{\boldsymbol{\Phi}_{i}\right\}_{i=1 . . n-1}$. Expression (10) suggests that $m_{0}$, as a difference between a "static" mass $m_{s t}$ (the one involved in the static mode) and the masses $\left\{m_{i}\right\}_{i=1 . . n-1}$, may be viewed as a residual mass. Expression (12) justifies the name "antioscillator": the natural frequency of the $i$ single-dof system $(i>1)$ depicted in Fig. 2 is an antiresonant frequency of the structure.

The AO model is an extension to the ones proposed by Skivakumar [3] and Abrate [2]: these latter model truncate all the $n-1$ antioscillators and keep only the "static stiffness" and the residual mass.

\section{The sphere-sphere impact}

Before considering a "soft" target impacted by a "rigid" projectile, in this section an impact between to hard bodies was analyzed. More precisely, in this section a steel sphere of radius $R$ at rest is struck by a similar sphere with an incident velocity $V_{0}=1 \mathrm{~ms}^{-1}$. The sphere characteristics are listed in table 1.

A finite element model was carried out for each sphere: tetrahedral elements having quadratic 


\begin{tabular}{cccc}
$f_{1}(\mathrm{~Hz})$ & $f_{2}(\mathrm{MHz})$ & $f_{3}(\mathrm{MHz})$ & $f_{4}(\mathrm{MHz})$ \\
\hline & & & \\
0 & 0.13 & 0.14 & 0.18 \\
\hline
\end{tabular}

Table 2: First eigenfrequencies of the sphere

interpolation, ten nodes and three degrees of freedom at each node were considered. The mesh was rather coarse: 1053 nodes (i.e. 3159 dof) and 602 elements were used. Then a mass matrix $\left(\mathbf{M}_{i}\right)$ and a stiffness matrix $\left(\mathbf{K}_{i}\right)$ were built for each $i$ sphere $(i=1$ or 2$)$; the dof vector was $\mathbf{X}_{i}$. Table 2 gives the "first" eigenfrequencies. Obviously these frequencies may be multiple: for instance, six eigenmodes have a frequency equals to zero as there are six rigid body modes.

The interaction between both spheres was described by a Hertz law: the Hertz stiffness was:

$k_{H}=\frac{4}{3} \frac{E_{S}}{2\left(1-\nu_{S}^{2}\right)} \sqrt{\frac{R}{2}}=10^{10} \mathrm{Nm}^{-3 / 2}$

Only one dof per sphere was supposed to interact with the other sphere: the $i_{1_{0}}$ dof for the first sphere and the $i_{2_{0}}$ dof for the second one. Accordingly the first sphere acted upon the second sphere and the interaction force was:

$\mathbf{F}_{H}=k_{H}\left(\mathbf{X}_{i_{2}}-\mathbf{X}_{i_{0}}\right)^{3 / 2}$ when $\quad \mathbf{X}_{i_{2_{0}}}>\mathbf{X}_{i_{1_{0}}}$

Each sphere was described with an AO model as well: the dof of interest was obviously the interaction dof $i_{0}$ for each sphere. The dof vector was then $\lambda_{i}$ and the interaction force was written:

$\mathbf{F}_{H}=k_{H}\left(\lambda_{i_{0}}-\lambda_{i_{1_{0}}}\right)^{3 / 2}$ when $\quad \lambda_{i_{2_{0}}}>\lambda_{i_{1_{0}}}$

The impact event was simulated with the full model simulation, with a modal expansion, and 
with an AO formulation.

\subsection{Simulations}

\section{Full Model}

The $2 \times 3159$ dof were considered. The equations were solved with a $\beta-\gamma$ Newmark algorithm coupled with a Newton-Raphson procedure to account for the nonlinearity. The impact force is reported in Figure 3. It can be noted that the maximum of the force was 811.5 $\mathrm{N}$ and the impact duration was $72 \mu \mathrm{s}$.

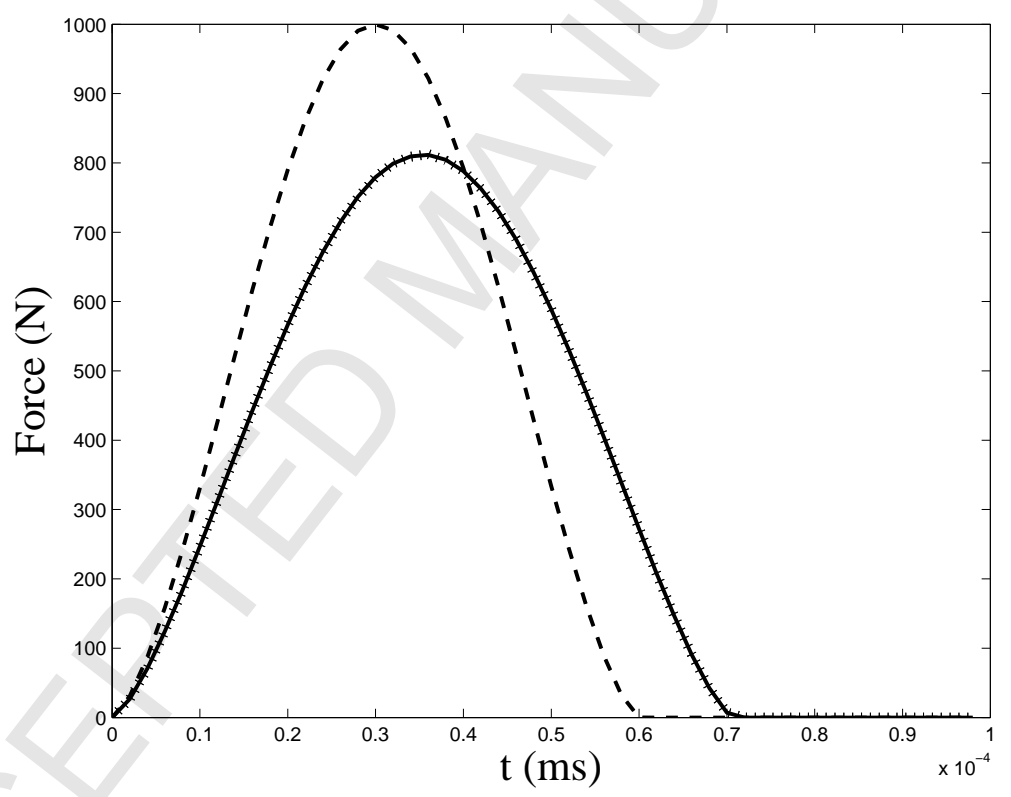

Fig. 3. Impact force: Full model (-); rigid mass (- -) (1-dof); AO model ( ...) (2-dof)

The AO simulation

The numerical cost to determine the AO model was due to the computation of the eigenvectors. Fortunately, few eigenvectors were enough: in the following only one AO will be considered. The characteristics of the model are given in Table 3. 


\begin{tabular}{cccc}
$k_{0}\left(\mathrm{Nm}^{-1}\right)$ & $m_{0} / m_{\text {sphere }}(\%)$ & $k_{1}\left(\mathrm{MNm}^{-1}\right)$ & $m_{1} / m_{\text {sphere }}(\%)$ \\
\hline 0 & 0.10 & 275.7 & 99.90 \\
\hline
\end{tabular}

Table 3: AO model of the sphere

This method is the classical one and has been widely recommended since the researches done by Timoshenko. Indeed, this method has given interesting results for structures; for solids the interest may be not so obvious, as shown in Figure 4. Indeed the convergence is very slow: even for 1000 modes (i.e. one third of the total number of dof), the impact force is over-estimated by $7 \%$ and the impact duration is underestimated by $5 \%$. Obviously these differences are not large but the results are definitively not interesting regarding the computational cost required to determine 1000 modes: the full model provides the result with much less CPU time.

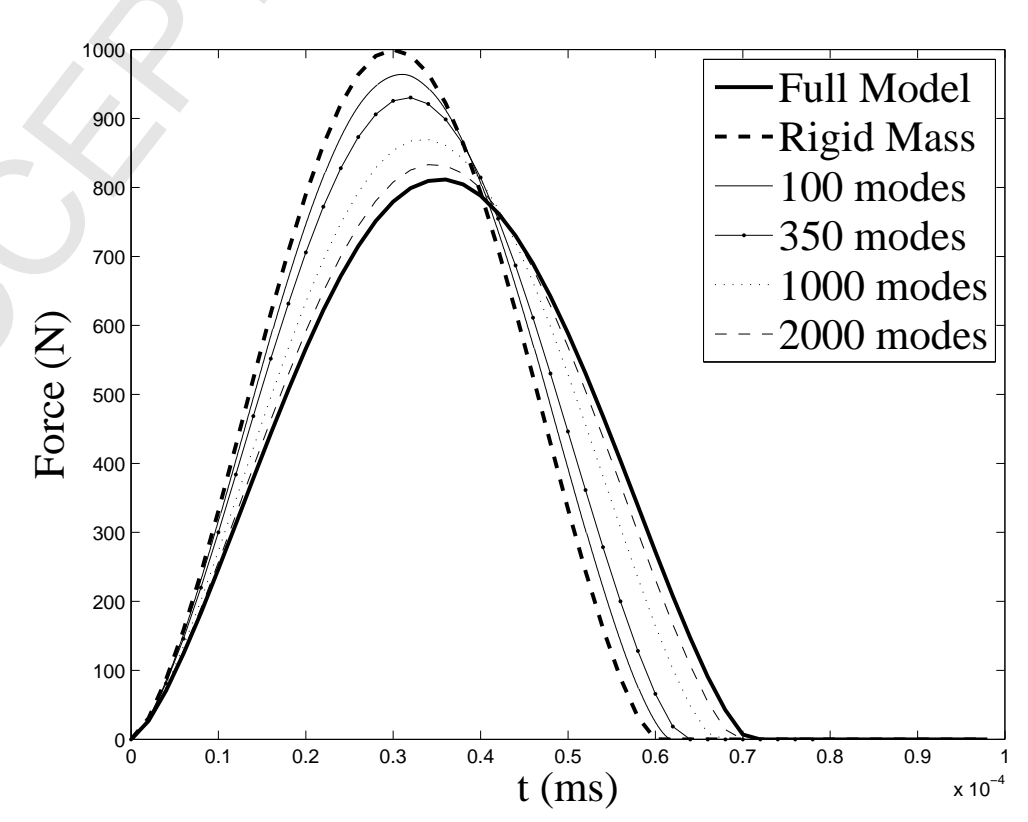

Fig. 4. Impact force evolution with respect to the number of modes 


\subsection{Discussion}

Figure 3 shows that two dof per sphere are enough to model a sphere-sphere impact. Indeed, the 1-AO model provides the same impact force than the full sphere model which requires 3159 dof per sphere. The same figure shows that the rigid mass model associated to the Hertz law overestimates the force by $23 \%$ and underestimates the impact duration by $16 \%$.

Considering the AO parameters (see Table 3), a striking result is obtained: most of the mass $(99.9 \%)$ is included in the first AO mass. That does not mean that the rigid mass model is then validated. Indeed, Figure 3 shows that the maximum of the rigid mass model impact force is $999.9 \mathrm{~N}$ whereas the maximum of the 1-AO model impact force is $812.6 \mathrm{~N}$; the impact duration is different in both cases as well. Accordingly the mass $m_{0}$ and the stiffness $k_{1}$ play a great role in the impact event. However, Figure 5 shows that the nonlocal deformation energy (i.e. stored in stiffness $k_{1}$ ) of the sphere initially at rest, as well as the local deformation energy (i.e. stored in the Hertz stiffness) are negligible compared to the kinetic energy, just after the impact: the initial kinetic energy is totally transferred to the other sphere. Then, no "dissipation" due to residual vibrational energy occurred. In other words, as $m_{1}$ is almost equal to the sphere mass, the impact between two spheres may be modelled as a rigid mass impact but the Hertz interaction law must be modified to recover the impact characteristics (maximum of the force, impact duration). This modified interaction law must include the $m_{0}$ and $k_{1}$ parameters (see Figure 6 ); consequently, the initial velocity has an influence on the interaction law, due to $m_{0}$ as indicated by Figure 7. This Figure shows that for small impact velocities, the indentation is limited and then the interaction depends only on the Hertz nonlinear stiffness: the displacements of mass $m_{0}$ and the rigid mass are almost the same, so both masses may be gathered. Indeed, for a $10^{-4}-\mathrm{ms}^{-1}$-impact velocity leads to a local deformation energy 200 times greater than the nonlocal 
deformation energy: this confirms that spring $k_{1}$ has almost no deformation. On the contrary, for higher impact velocities both deformation energies tend to be the same, so no spring can be considered as rigid compared to the other one: mass $m_{0}$ can not be gathered to the rigid mass anymore. This explains why a modified interaction law is needed for higher impact velocities.

The difference between the 1-dof model (rigid mass model) and the 2-dof model (1 AO model) must be addressed. Recall that an impact event involves two main phenomena: a local deformation due to the contact between both spheres and an nonlocal deformation due to the wave propagation. The Hertz nonlinear stiffness represents the local deformation: then, when the rigid mass model is used, the nonlocal deformations of the spheres are disregarded. On the contrary, the $1 \mathrm{AO}$ model allows for the wave propagation through mass $m_{0}$ and stiffness $k_{1}$ : particularly, there is no simultaneity between the beginning of the impact and the movement of mass $m_{1}$. This is confirms by Fig. 5 which shows that the sphere at rest stored deformation energy during the impact and released it almost totally at separation. So, mass $m_{0}$ seems negligible as it represents only $0.1 \%$ of the total mass, but it plays a great because it makes possible the wave propagation effect.

\section{Beam-sphere impact}

The last section highlighted the difference between a 1-dof model (the rigid mass model) and a 2-dof model (the 1-AO model) for the sphere-sphere impact. This section aims to address the influence of the sphere model on the response of the impacted structure.

The projectile was the sphere studied in the previous section (cf Tables 1 and 2). The finite element model, and then the OA model, were the same as well. 


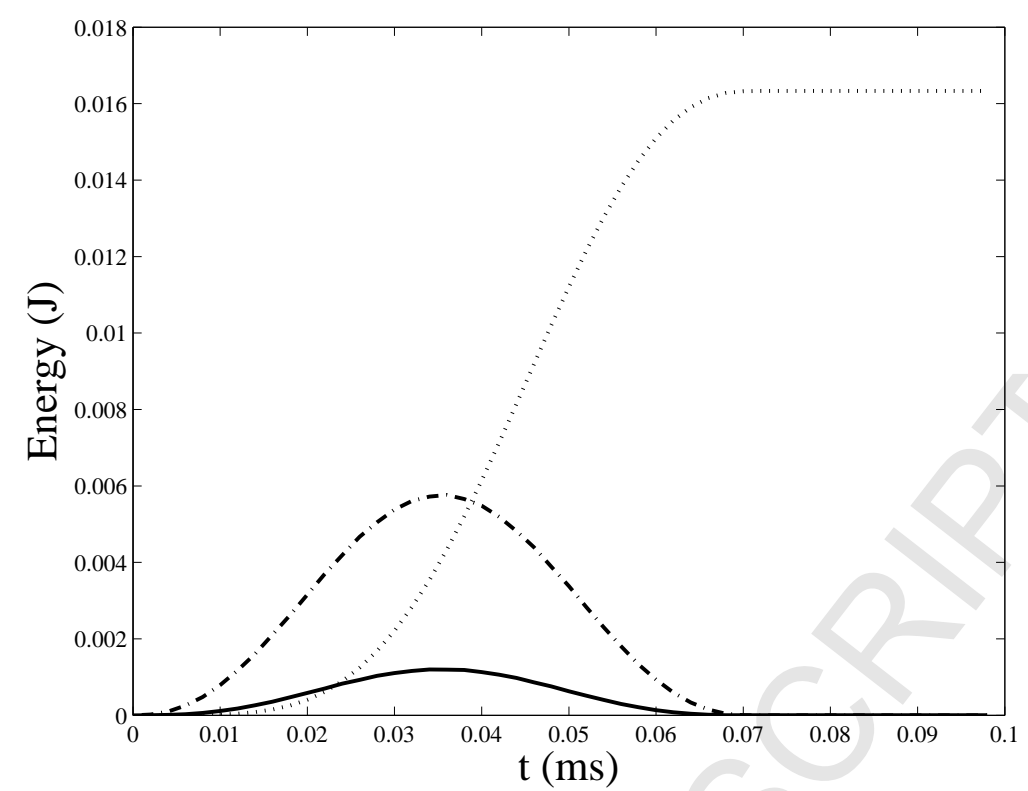

Fig. 5. Sphere initially at rest: local deformation energy $(\cdot-\cdot)$; nonlocal deformation energy $(-)$; kinetic energy $(\cdots)$

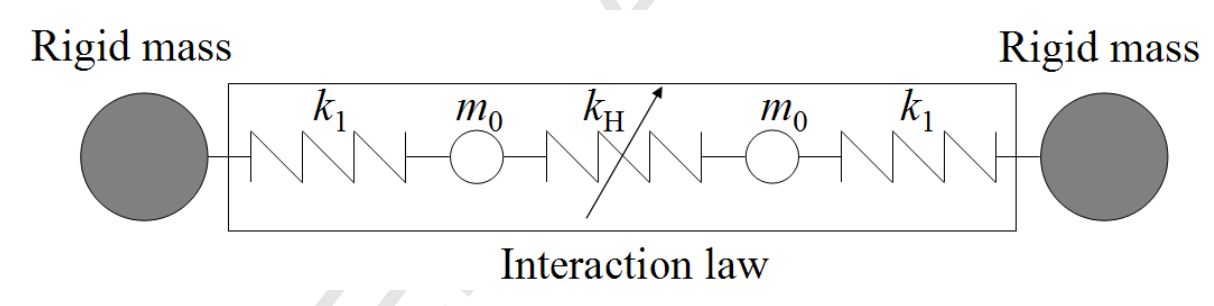

Fig. 6. Sphere-Sphere impact model: rigid masses and interaction law

This target was a simply supported aluminum beam (see its characteristics in Table 4) modelled with hexahedron finite elements; the elements were defined by eight nodes having three degrees of freedom at each node: translations in the three nodal directions. The mesh was coarse: 459 nodes and 200 elements were required to model the beam. The impact occurred at the center of the top surface. The AO were determined following the procedure described in section 2: the dof associated with the AO model is the beam impact dof.

To simulate the beam-sphere interaction, the Hertz was used; the Hertz stiffness was then:

$k_{H}=\frac{4}{3} E^{*} \sqrt{R}$ 


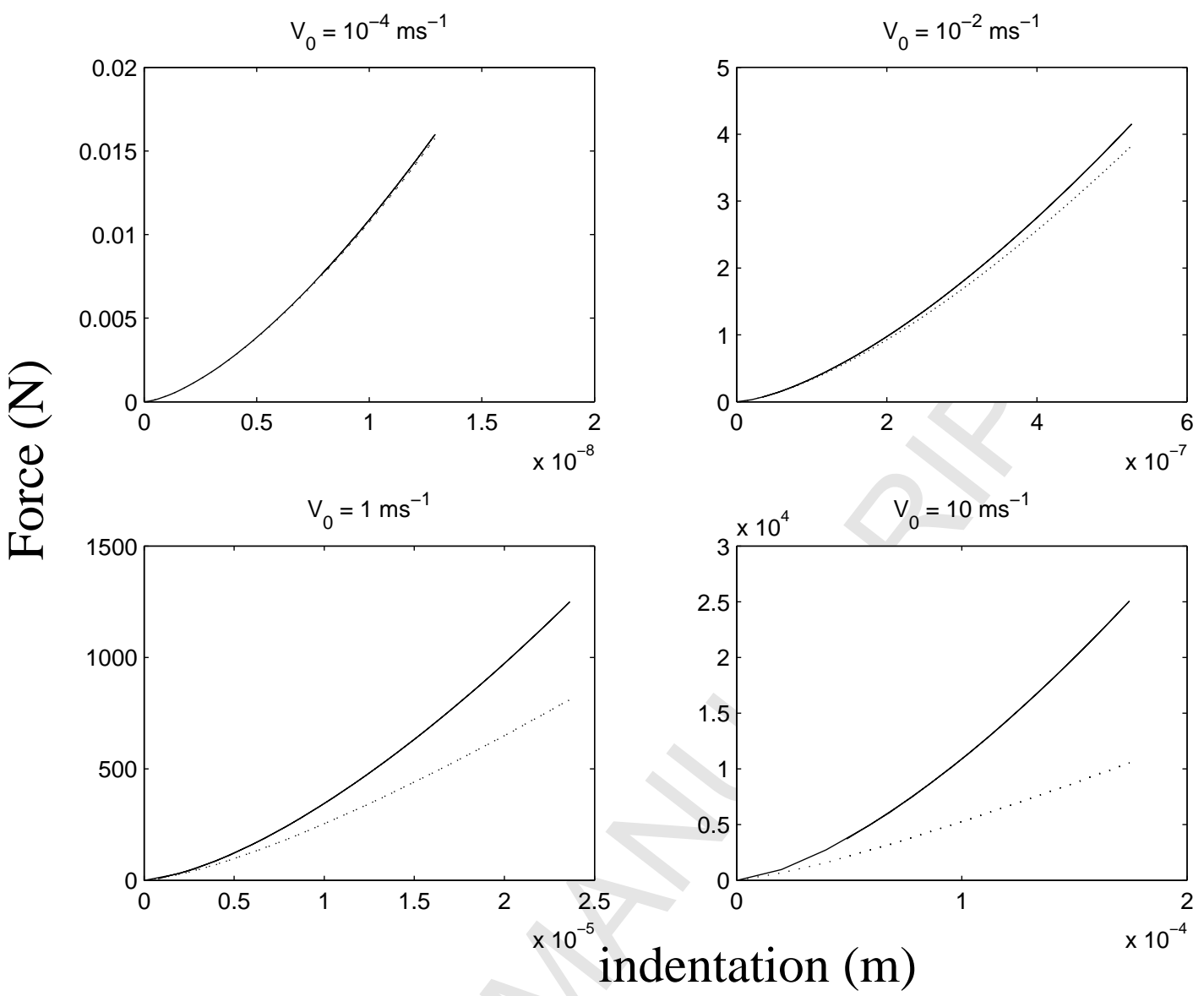

Fig. 7. Indentation vs Interaction Force for several initial velocities: Hertz interaction law (-); Modified interaction law $(\cdots)$

\begin{tabular}{cccccc}
$E_{B}(\mathrm{GPa})$ & $\nu_{B}$ & $\rho_{B}\left(\mathrm{kgm}^{-3}\right)$ & $L(\mathrm{~mm})$ & width $(\mathrm{mm})$ & height $(\mathrm{mm})$ \\
\hline 70 & 0.3 & 2400 & 1000 & 20 & 20 \\
\hline
\end{tabular}

Table 4: Characteristics of the beam

with:

$\frac{1}{E^{*}}=\frac{1-\nu_{B}^{2}}{E_{B}}+\frac{1-\nu_{S}^{2}}{E_{S}}$

\section{Simulations}

First, a full model simulation was carried out. The time step was $2 \mu$ s and the duration was 


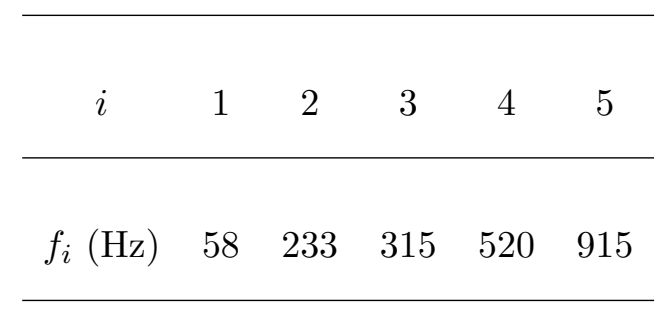

Table 5: First eigenfrequencies of the beam

$200 \mu \mathrm{s}$. The first eigenfrequencies of the beam are listed in Table 5 . Tables 2 and 5 show that the sphere first eigenfrequencies were much higher than the beam ones.

Then AO model simulation was performed: it had been found that $1 \mathrm{AO}$ for the sphere and 29 dof for the beam were required to obtain the full model simulation results (displacements and impact force). Then, once again, this proves the efficiency of the AO model to simulate an impact event: a 29-dof model is able to give the same results than a 1362-dof model for the beam; as to the sphere, the benefit is even more striking, as shown in the previous section. So, in the following, beam will be model with $28 \mathrm{AO}$; however, the AO number for the sphere must be addressed.

It may be surprising that $28 \mathrm{AO}$ were required for the beam whereas $1 \mathrm{AO}$ was enough for the sphere but this fact may be explained by comparing Table 3 and Figure 8. Indeed, the 28-AO beam masses represented $98 \%$ of the static mass $m_{s t}=467 \mathrm{~g}$, whereas 1 -AO sphere mass represented $99.9 \%$ of the sphere mass. More precisely, the first AO beam mass represented only $53 \%$ of $m_{s t}$ : thus the dynamic of the beam was not able to be described well with one (or few) AO. 


\section{ARTICLE IN PRESS}

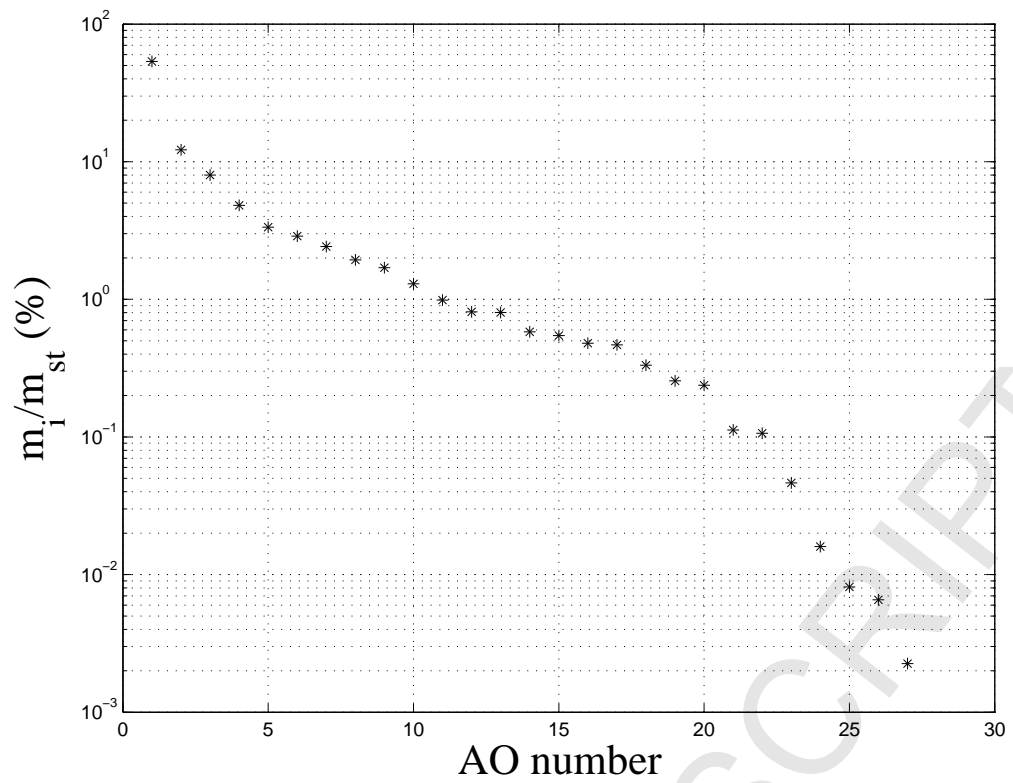

Fig. 8. Beam AO masses

Discussions

Figures 9 and 10 show the impact force and the displacements of the beam and sphere center of mass obtained with the rigid mass model and with the 1-AO model. Once again, the impact force is overestimated with the rigid mass model and the impact duration underestimated. The sphere displacement is not perfectly described, whereas the beam displacement is rather good. Nevertheless, Figure 10 reports the beam displacement during the first $200 \mu \mathrm{s}$, that was short with respect to the beam first period: the beam maximum displacement was only $25 \mu \mathrm{m}$. Hence longer simulation was carried out during $20 \mathrm{~ms}$ for both models. Figure 11 shows that the displacement was the same for both model. This fact shows that the impact force is not the most important characteristic of an impact event to design a structure: hence the quality of a model must be assessed by its ability to provide the response of the structure, rather than its ability to provide the impact force. 


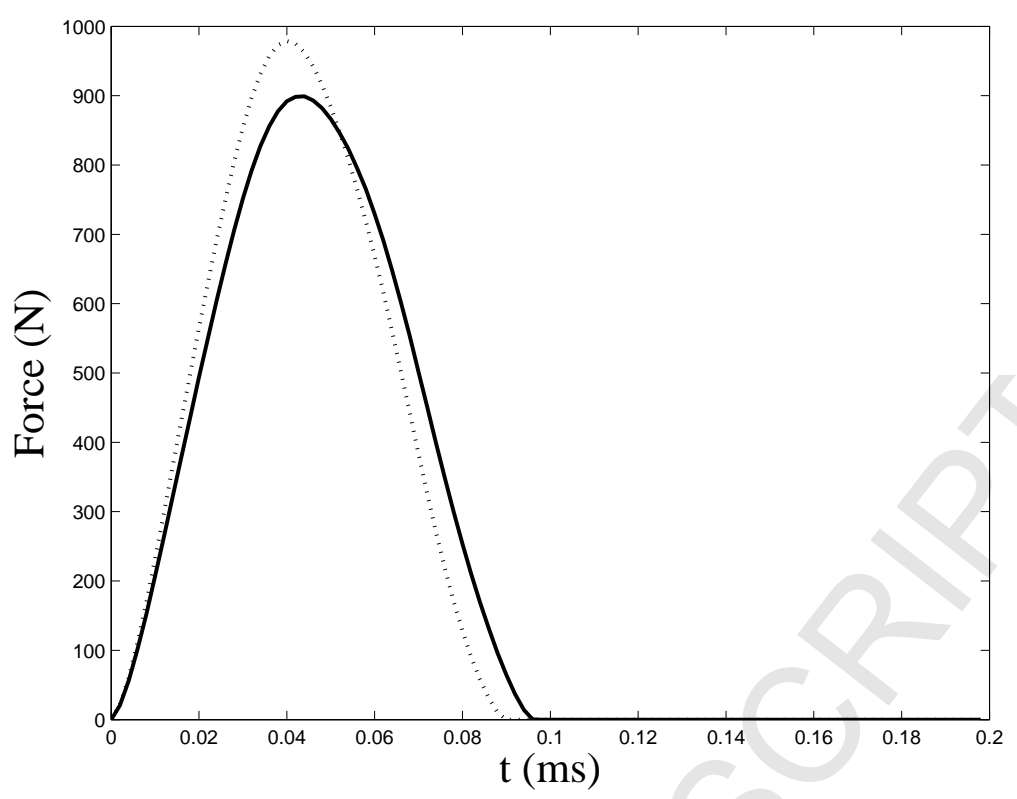

Fig. 9. Impact force: 1-AO model $(-)$; rigid mass model $(\cdots)$

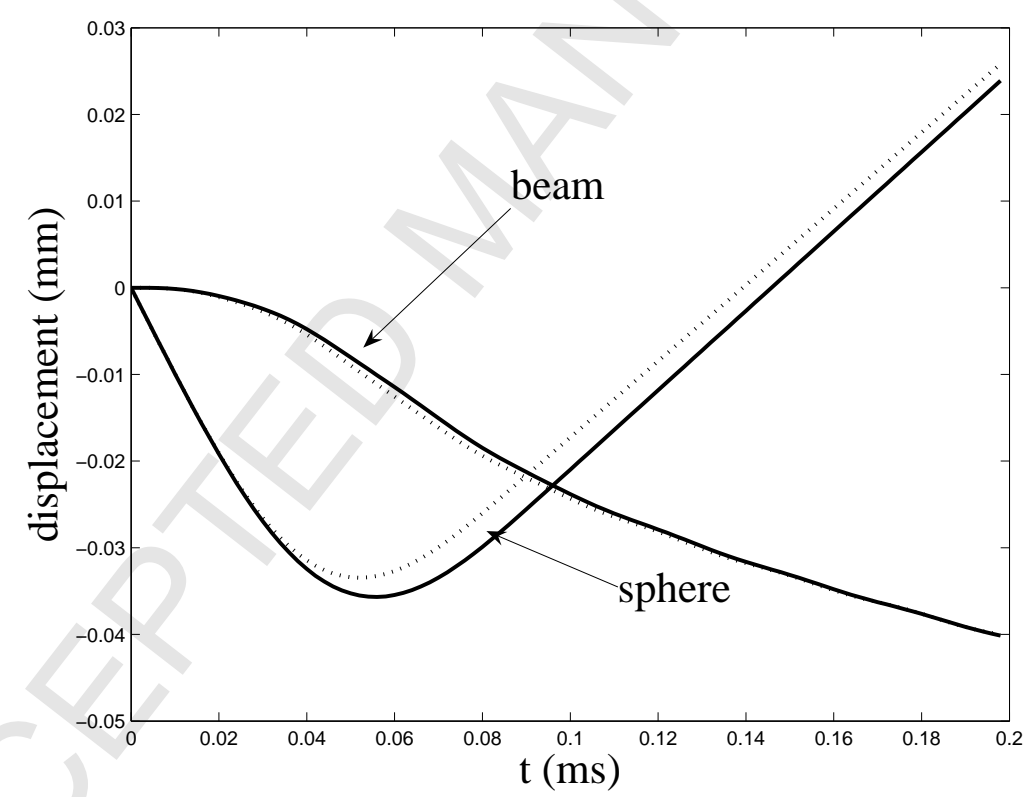

Fig. 10. Sphere and beam center of mass displacement: AO model (-); rigid mass model $(\cdots)$

\section{Conclusion}

This paper addressed the problem of modelling a projectile which has eigenfrequencies much higher than the target eigenfrequencies. Usually, the projectile is described by a 1-dof system (a rigid mass) and the interaction is modelled by an interface law such as the Hertz law.

However, the study of a sphere-sphere impact showed that the rigid mass model was not able 


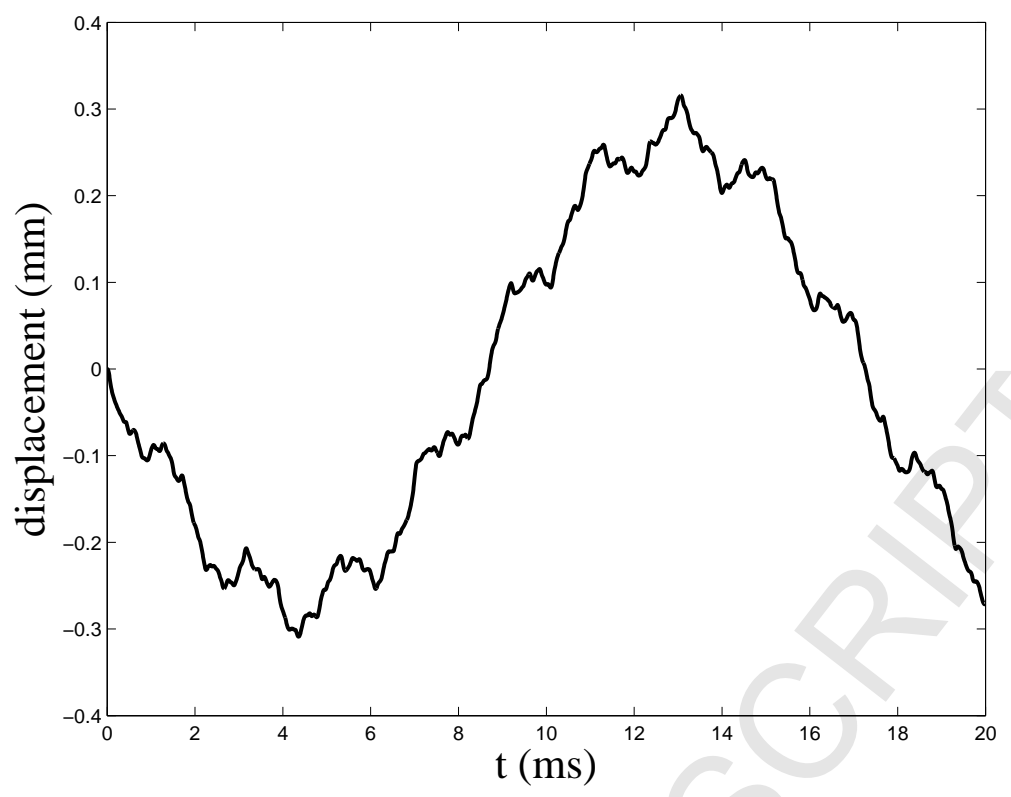

Fig. 11. Beam gravity center displacement: $1-\mathrm{AO}$ model and rigid mass model (-) to obtain the impact force whereas a 2-dof model may be suitable. In fact, this 2-dof model may be viewed as a rigid mass associated with a modified Hertz law.

The same conclusion was made when studying the impact of a sphere upon a structure, a beam modelled in 3D: to obtain the impact force the projectile must be modelled by a 2-dof system (1-AO) at least; the beam was modelled with a 28 AOs. Nevertheless, when designing a structure, the structural response (displacement) is must more important than the impact force: in that case, the rigid mass model model is relevant.

Then to model a projectile by a rigid mass may be definitely correct even if the obtained impact force is not estimated well: the same structural response may be obtained for different impact forces.

\section{REFERENCES}

[1] S. Abrate. Impact on Composite Structures. Cambridge University Press, 1998.

[2] S. Abrate. Modeling of impacts on composite structures. Composite Structures, 


\section{ARTICLE IN PRESS}

$51: 129-138,2001$.

[3] K.N. Shivakumar, W. Elber, and W. Illg. Prediction of impact force and duration due to low-velocity impact on circular composites laminates. Journal of Applied Mechanics, 52:674-680, September 1985.

[4] K.Q. Wu and T.X. Yu. Simple dynamic models of elastic plastic structures under impact. International Journal of Impact Engineering, 25:735-754, 2001.

[5] W. Goldsmith. Impact. Edward Arnold publishers Ltd, 1960.

[6] W.J. Stronge. Impact mechanics. Cambridge University press, 2000.

[7] E. Jacquelin, J.P. Lainé, A. Bennani, and M. Massenzio. A modelling of an impacted structure based on constraint modes. Journal of Sound and Vibration, 301 (3-5):789-802, April 2007.

[8] E. Jacquelin, J.P. Lainé, A. Bennani, and M. Massenzio. The anti-oscillator model parameters linked to the apparent mass frequency response function. Journal of Sound and Vibration, 312 (4-5):630-643, May 2008.

[9] S. Pashah, M. Massenzio, and E. Jacquelin. Structural response of impacted structure described through anti-oscillators. International Journal of Impact Engineering, $35: 471-486,2008$. 